\title{
The Impact of Design on Feelings of Trust of Online Information for Family Caregivers of People with Dementia
}

\author{
Thana Hussein \\ Systems Design Engineering, \\ University of Waterloo \\ 200 University Ave W \\ Waterloo, Ontario Canada N2L 3G1 \\ tghussei@uwaterloo.ca
}

\author{
Jennifer Boger \\ Systems Design Engineering, \\ University of Waterloo \& \\ Research Institute for Aging \\ 200 University Ave W \\ Waterloo, Ontario Canada N2L 3G1 \\ jboger@uwaterloo.ca
}

\author{
Frank Rudzicz \\ Computer Science, \\ University of Toronto \\ 550 University Avenue \\ Toronto, Ontario Canada M5G 2A2 \\ frank@spoclab.com
}

\begin{abstract}
CARE-RATE is an online assistive-tool being created to support caregivers in searching for information that fits their individualized needs. As the use of the web for information continues to expand, the issue of how to trust online information is a key concern for caregivers of people with dementia. Several studies have been conducted exploring how website design affects trust, however, no work has been done for interface design for caregivers of people with dementia. This research explores what design features may significantly impact trust for family caregivers of people with dementia. 20 at-home caregivers will be asked to complete a set of search-based tasks through CARE-RATE. Half of the participants will be exposed to a standard search engine results page (SERP), while the other half will be given a SERP with additional information such as ratings from other caregivers, transparency of the author/organization, and images associated with the content. The data derived from this study will be consolidated into a modified Lens Model to further understand how cues impact source selection and trust and, subsequently, integrated into CARERATE's interface design.
\end{abstract}

Family caregiver, dementia, online information, trust, interface design, UI/UX

\section{INTRODUCTION}

There are approximately 8.5 million family members who provide care at home for persons with dementia (Fulmer, 2017). With the number of people living with dementia expected expected to rise to 16 million by 2050 (Alzheimer's Association, 2017); with no cure and an increasing aging global population, the number of caregivers is expected to continue to rise as well. The multitude of tasks and responsibilities related to being a family caregiver for someone living with dementia can become difficult or overwhelming. The preponderance of people living with dementia receive care from family members who are entrusted with fulfilling this challenging role. While there is a plethora of information available online, caregivers may not be able to find it (Peterson et al., 2016). The number of social media, internet sites, and mobile applications that offer resources and information is doubling in size every two years with the cumulative amount of data expected to increase to 44 zettabytes by 2020 (Turner et al., 2014). Finding reliable information online requires an individual to be meticulous in how he or she is searching for information; however, this requires time, effort, and knowledge of how to perform an effective search, all of which can be especially problematic for overburdened caregivers. Moreover, it can be difficult for caregivers to ascertain the legitimacy of the sources that they find.
To address this need we are creating CARE-RATE, which is an online tool that uses natural language processing and artificial intelligence to search the web for products, strategies, and local resources using context-specific information to fit results to individualized needs. Part of this is creating a user interface that is designed to support caregiver abilities and preferences. Previous focus groups we held with caregivers highlighted their need for being able to identify information trusted sources of information (Boger et al., 2017; Polgar, Rudzicz, \& Boger, 2016). Little work has been done for interface design for caregivers and, to our knowledge, no research has been done to explore what cues impacts trust and source selection. This work seeks to bridge this gap by systematically investigating design elements of a search engine results page (SERP) to support the ability of family caregivers of people living with dementia to ascertain their level of trust in the search results more easily.

\section{LITERATURE REVIEW}

\subsection{Caregivers and online information seeking}

Using conventional formal services and resources can be difficult for caregivers due to time and financial constraints (Beeber et al., 2008; Powell et al., 2011; Toseland et al., 1999). Kim et al. (2017) 
showed how family caregivers commonly use the internet to seek health information regarding dementia and how to effectively care for loved ones. Attributes of the internet that are significant in delivering resources and services include interactive traits that enable users to become more proactive as consumers of health care and internet-based healthrelated resources that can support informal caregivers in a cost-effective and sound manner.Internet-based care modality attributes to healthier well-being status (Marziali \& Garcia, 2011) and higher-levels of confidence in decision-making, self-efficacy, as well as an overall more positive demeanor towards providing care (Beauchamp et al., 2005; Lewis et al., 2010; Powell et al., 2008). However, caregivers with limited health literacy or ehealth literacy, such as individuals who are 65 years or older, men, African American, Hispanic, nonEnglish speakers, have lower incomes, and/or those with less than secondary education (Cutilli \& Bennett, 2009) experience disparities in health and access to health care resources (Sudore et al., 2006) - including online ones - which may inhibit their ability to make opportune decisions. Indeed, the internet continues to figure prominently in people's quotidian lives, especially pertaining to the delivery of healthcare interventions and services, however, it is imperative to consider the additional information caregivers seek online beyond healthrelated regimens.

To better understand these needs and the ways in which they could best benefit from the CARE-RATE technology, we conducted two focus groups with caregivers from the community (Boger et al., 2017; Polgar, Rudzicz, \& Boger, 2016). Our focus groups identified three key areas where caregivers most desire more online support: being able to learn more about a specific topic, finding strategies and advice from trusted sources to help them with a current concern, and connecting with other caregivers.

\subsection{Trust and web design}

Designing for trust in technology-mediated interfaces is of increasing importance in human-computer interaction (Riegelsberger, Sasse, \& McCarthy, 2005). Vital dimensions of credibility for both offline and online trust include expertise, as many caregivers have limited knowledge of the situation, which is why they turn to online expertise to augment knowledge he or she lacks (Hankowski et al., 1994; Kantowitz et al., 1997; Lee \& Moray, 1992; Shelat \& Egger, 2002). Website design elements such as layout, color, and navigation all impact trust. Websites should be visually appealing, meaning they feature a good use of color and graphics text balance and should make a good utilization of visual design elements (Kim \& Moon, 1997), such as incorporating professional images of products (Nielsen et al., 2000) and exhibiting content that is free from any typographical and grammatical errors (Fogg et al., 2001; Nielsen et al., 2000). Furthermore, individuals will likely trust a professional looking website that renders it easy to search for information (Nielsen et al., 2000) as well as carrying out transactions (Lohse \& Spiller, 1998; Nielsen et al., 2000). Caregivers and other users may find sites more trustworthy if they feature a third-party trust logo, although studies (Nielsen et al., 2000) have shown that users often either do not notice the presence of such logos or do not care, possibly because they do not find any currency in them due to the ubiquity of websites that disseminate false information. Reputation systems (recommender systems, collaborative filtering, or social navigation) aid in trust arbitrations (Dieberger et al., 2000; Resnick \& Varian, 1997; Resnick et al., 2000). In information seeking and conventional online transactions, aggregated ratings provide a meaningful history that can be used by other people to judge the risk of a transaction or the value of information from a given provider. Research has suggested that trust in a mechanized recommender can be increased by a colloquial interface and disclosure of what the recommender system knows about the user (Zimmerman \& Kurapati, 2002). Finally, a website's ease of use often influences its credibility since the visitor would perceive that website as being more predictable and honest. Caregivers who are seeking information who find that a website is easy to use will experience a lower cognitive burden and thus will have more cognitive resources that he or she must attend to credibility cues (Cheskin Research \& Studio Archetype/Sapient, 1999; Nielsen et al., 2000)

Perception of credibility further influences perception of risk, so this inverse relationship means that if a website is viewed as extremely credible, then it can be assumed that interacting with it will entail a lower risk (Corritore, Kracher, \& Wiedenbeck, 2003). As discussed in our focus groups, providing care for someone with dementia is a serious matter and they feel there is little or no room for error, therefore caregivers are extra vigilant about the websites they engage with; information content, navigation capabilities, and web design (including graphics and images) all play a role in whether or not the caregiver feels a website should be trusted or not. Pursuing information online and arbitrating credible sources, however, can be a rather challenging task, especially for dementia caregivers as they are one of the most vulnerable caregiver populations. Amidst this challenging task, dementia caregivers are encountering elevated levels of stress, care-giving intensity, and diverse health problems covering a range of physical, psychological, social, and spiritual facets (Family Caregiver Alliance, 2009; Schulz \& Sherwood, 2008; Zarit, 2006). Trust also incorporates the discernment that a person has vulnerabilities and those susceptibilities could be 
infringed or capitalized upon (Sabel, 1993). Vulnerability, with the simultaneous probability of exploitation, should likewise, be explored when designing a trustworthy interface for dementia caregivers (Deutsch, 1962; Mayer, Davis, \& Schoorman, 1995; Zand, 1972).

\section{CARE-RATE'S DESIGN}

\subsection{Wireframe testing}

The CARE-RATE interface is being built through several iterative stages of participatory design. Three potential CARE-RATE interface designs were evaluated as wireframes that were built upon website design literature and tested with eight caregivers through a multi-modal, semi-structured interview procedure to assess design features (Chauhan et al., 2018). 100\% of the participants rated the search bar (see Figure 1), as the most appealing and trustworthy-looking, with participants mentioning that they liked its simplicity and perceived ease of use. This aligns with a person's general proclivity to trust hinges on the user's prior experience with a similar situation or object of trust, therefore preference for the search bar design is likely heavily influenced by its similarity in appearance to other search engines on the internet. Participants also had strong opinions regarding the importance of the perception of trust toward online sites, which included navigational architecture, information content accuracy as determined by the credentials of the author and/or the organization sponsoring him or her, seals of approval from organizations (e.g., the Alzheimer Society), branding, and reputation all figure prominently. An input system, where caregivers can observe what works for and is trusted by other caregivers, is highly desired. The results from our focus groups and wireframe testing coupled with literature and an ethnographic exploration of existing websites, such as Amazon and Google, informed the prototype CARE-RATE interface design, which will be evaluated in this next phase of research by family caregivers living in the community.

\section{CARE-RATE}

CARE-RATE is an interactive system than can help you find information you need.

What can I help you with today?



Figure 1: CARE-RATE search bar wireframe design

\subsection{Trust study}

The objective of this in-progress study is to systematically understand trust-supporting design cues; these insights will be integrated into CARERATE's SERP design. The framework used for this study asses two distinct cognitive processes to form an assessment of trust for a website: an initial heuristic-based judgment of a website, followed by a more thorough and analytical evaluation of the information content. In this study, we will refer to the first stage (heuristic analysis) as "Tier 1," trust in the CARE-RATE user interface. The second stage (evaluation of information content) will be referred to as "Tier 2," trust in the information and content CARE-RATE provides. 20 at-home caregivers will be asked to complete a set of search-based tasks through CARE-RATE in oneon-one sessions. Representative tasks, informed by our previous research with focus groups and our advisory committee, will be used for testing. 10 participants will be in the control group in which they be given a baseline SERP layout, a design derived from our previous ethnographic study of comparable websites. The other 10 participants in the experimental group will be exposed to an altered CARE-RATE SERP that contains additional cues such as ratings from other caregivers, transparency of the author/organization providing the information, and images associated with the article. The study will be broken down into three segments: trust in Tier 1 rated on a 7-point Likert scale; trust in Tier 2 where participants will be asked to identify the cues that impacted their decision in source selection and to rate the significance of each cue on a 7-point Likert scale; and their overall trust of CARE-RATE (Tier $1+$ Tier 2) rated on a 7-point Likert scale once they have completed the tasks. Participants will be asked to talk-aloud throughout each task so we can futher understand their decision-making process. 




(a)

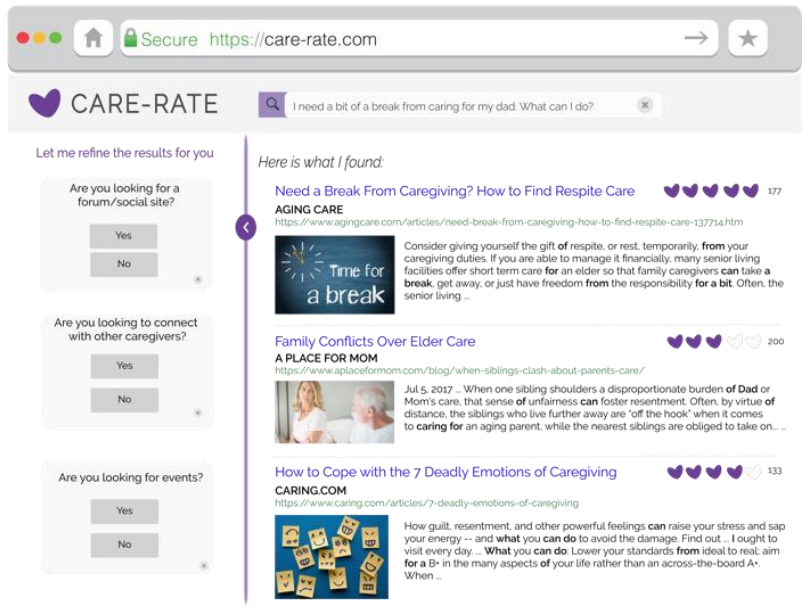

(b)

Figure 2: CARE-RATE's SERP. (a) control and (b) experimental.

The data derived from this study will be consolidated into a modified Lens Model (Figure 3). The Lens Model is a way of thinking about describing the relations between the environment and the behavior of organisms in the environment (Wolf, 2005) The fundamentals were developed by Egon Brunswik (1952) and popularized by Kenneth Hammond through research on social judgments and how individual's use information from the task environment to make clinical judgments (Hammond, 1955). By looking at the correlations between the design cues and the judgment (source selection and trust), we can get some idea of the "importance" of the cues to judge and furthermore, how Tier 2 trust impacts Tier 1 trust. Furthermore, this research will allow us to better answer our question, that is, what design elements on a SERP significantly impact trust for family caregivers of people living with dementia, insight which be implemented into CARE-RATE to support caregivers in making trustworthy, informed decision.



Figure 3: Modified Lens Model (Dhami et al., 2004)

\section{CONCLUSION}

The data extracted from our focus groups with caregivers of people living with dementia and testing of wireframes with our advisory committee have shown that caregivers struggle with finding strategies and advice from trusted sources. These desires coupled with website design literature, form the basis of the CARE-RATE, an online intelligent tool aimed to assist family caregivers of people with dementia. In this paper we have presented a method for understanding the components of website design elements, such as transparency of author/organization, ratings from other caregivers, and images, influence trust for caregivers of people. These findings are the basis of the next stage of CARE-RATE's interface, which will be further evaluated by family caregivers living in the community.

\section{ACKNOWLEDGEMENTS}

We thank our advisory committee for their ongoing input and support of this work. This work was supported by AGE-WELL NCE Inc., a national research network supporting commercialization, knowledge mobilization and capacity building activities in technology and aging. AGE-WELL is a member of the Networks of Centres of Excellence (NCE), a Government of Canada program that funds partnerships between universities, industry, government and not-for-profit organizations. 


\section{REFERENCES}

Alzheimer's Association. (2017) Alzheimer's Disease Facts and Figures,.13(4), 325-373. doi:10.1016/j.jalz.2017.02.001

Beauchamp, N., Irvine, A. B., Seeley, J., \& Johnson, B. (2005) Worksite-Based Internet Multimedia Program for Family Caregivers of Persons with Dementia. The Gerontologist, 45(6), 793-801. doi:10.1093/geront/45.6.793

Beeber, A. S., Thorpe, J. M., \& Clipp, E. C. (2008) Community-Based Service Use by Elders with Dementia and Their Caregivers. Nursing Research, 57(5), 312-321. doi:10.1097/01.nnr.0000313500.07475.eb

Boger, J., Rudzicz, F., Chinaei, H., Jónasdóttir, S. K., Wambua, M. \& Polgar, J. (2017) CARERATE: Initial Development of an Artificially Intelligent Online Tool for Connecting Caregivers to Relevant Support. Rehabilitation Engineering and Assistive Technology Society of North America (RESNA), New Orleans, LA, June 2830.

Brunswik, E. (1952) The Conceptual Framework of Psychology. International Encyclopedia of Unified Science, 1(10), Chicago: The University of Chicago Press.

Chauhan, P., Boger, J., Hussein, T., Moon, S., Rudzicz, F. \& Polgar, J. (2018) Creating the CARE-RATE Interface Through Multi-Modal Participatory Design with Caregivers of People With Dementia. 11th World Conference of the International Society for Gerontechnology (ISG2018), St. Petersburg, FL, May 7-11.

Cheskin Research \& Studio Archetype/Sapient. (1999) E-Commerce Trust Study. http://www.cheskin.com/p/ar.asp? mlid=7\&arid=4 0\&art=0\&isu=1 (April 2018)

Corritore, C. L., Kracher, B., \& Wiedenbeck, S. (2003) On-line trust: Concepts, Evolving Themes, a Model. International Journal of Human Computer Studies, 58(6), 737-758. doi:10.1016/S1071-5819(03)00041-7

Cutilli, C. C., \& Bennett, I. M. (2009) Understanding the Health Literacy of America Results of the National Assessment of Adult Literacy. Orthopaedic Nursing / National Association of Orthopaedic Nurses, 28(1), 2734. doi:10.1097/01.NOR.0000345852.22122.d6

Dhami, M. K., Hertwig, R., \& Hoffrage, U. (2004) The Role of Representative Design in an Ecological Approach to Cognition. Psychological Bulletin, 130, 959-988. doi:10.1037/00332909.130.6.959
Deutsch, M. (1962) Cooperation and Trust: Some Theoretical Notes. In M. R. Jones

(Ed.), Nebraska Symposium on Motivation, 275320. Oxford, England: University of Nebraska Press.

Dieberger, A., Dourish, P., Höök, K., Resnick, P., \& Wexelblat, A. (2000) Social Navigation:

Techniques for Building More Usable Systems. Interactions, 7(6), 36-45. doi:10.1145/352580.352587

Family Caregiver Alliance. (2006) Caregiver Health. https://www.caregiver.org/caregiver-health (April 2018)

Fulmer, T. (2017) Making Dementia Caregiving Support More Available to Those Who Need It. The John A. Hartford Foundation.

https://www.johnahartford.org/blog/view/making dementia-caregiving-support-more-available-tothose-who-need-it (April 2018)

Hammond, K. R. (1955) Probabilistic Functioning and the Clinical Method. Psychological Review, 62(4), 255-262. doi:10.1037/h0046845

Hammond, K.R. (1980) Introduction to Brunswikian Theory and Methods. New Directions for Methodology of Social and Behavioral Science, 3, 1-11.

Kim, H., Powell, M. P., \& Bhuyan, S. S. (2017) Erratum to: Seeking Medical Information Using Mobile Apps and the Internet: Are Family Caregivers Different from the General Public? Journal of Medical Systems, 41(4) doi:10.1007/s10916-017-0707-6

Lewis, M. L., Hobday, J. V., \& Hepburn, K. W. (2010) Internet-Based Program for Dementia Caregivers. American Journal of Alzheimers Disease \& Other Dementiasr, 25(8), 674-679. doi:10.1177/1533317510385812

Lohse, G. L., \& Spiller, P. (1998) Electronic Shopping. Communications of the ACM, 41(7), 81-87. doi:10.1145/278476.278491

Marziali, E., \& Garcia, L. J. (2011) Dementia Caregivers' Responses to 2 Internet-Based Intervention Programs. American Journal of Alzheimers Disease \& Other Dementiasr, 26(1), 36-43. doi:10.1177/1533317510387586

Mayer, R. C., Davis, J. H., \& Schoorman, F. D. (1995) An Integrative Model of Organizational Trust. The Academy of Management Review, 20(3), 709. doi:10.2307/258792

Nielsen, J., Molich, R., Snyder, C., \& Farrell, S. (2000) E-commerce User Experience: Trust. Nielsen Norman Group.

Peterson, K., Hahn, H., Lee, A. J., Madison, C. A., \& Atri, A. (2016) In The Information Age, Do Dementia Caregivers Get the Information They 
Need? Semi-Structured Interviews to Determine Informal Caregivers' Education Needs, Barriers, And Preferences. BMC Geriatrics, 16(1) doi:10.1186/s12877-016-0338-7

Polgar, J., Rudzicz, F., and Boger, J. (2016) CARERATE: An Online Resource System for Caregivers of Persons with Dementia. International Society for Gerontechnology (ISG), Nice, France, Sept 28-30.

Powell, J., Chiu, T., \& Eysenbach, G. (2008) A Systematic Review of Networked Technologies Supporting Carers of People with Dementia. Journal of Telemedicine and Telecare, 14(3), 154-156. doi:10.1258/jtt.2008.00301

Resnick, P., \& Varian, H.R. (1997) Recommender Systems. Communications of the ACM, 40(3), 56-58. doi:10.1145/245108.245121

Resnick, P., Kuwabara, K., Zeckhauser, R., \& Friedman, E. (2000) Reputation Systems. Communications of the ACM, 43 (12), 45-48. doi: $10.1145 / 355112.355122$

Sabel, C. F. (1993) Studied Trust: Building New Forms of Cooperation in a Volatile Economy. Human Relations, 46(9), 1133-1170. doi:10.1177/001872679304600907

Schulz, R., \& Sherwood, P. R. (2008) Physical and Mental Health Effects Of Family Caregiving. Journal of Social Work Education, 44(Sup3), 105-113. doi:10.5175/jswe.2008.773247702
Sudore, R. L., Mehta, K. M., Simonsick, E. M., Harris, T. B., Newman, A. B., Satterfield, S. , Rosano, C. , Rooks, R. N., Rubin, S. M., Ayonayon, H. N., \& Yaffe, K. (2006) Limited Literacy in Older People and Disparities in Health and Healthcare Access. Journal of the American Geriatrics Society, 54: 770-776. doi:10.1111/j.1532-5415.2006.00691.x

Toseland, R. W., Mccallion, P., Gerber, T., Dawson, C., Gieryic, S., \& Guilamo-Ramos, V. (1999) Use of Health and Human Services by Community-Residing People with Dementia. Social Work, 44(6), 535-548. doi:10.1093/sw/44.6.535

Turner, V., Gantz, J. F., Reinsel, D., \& Minton, S. (2014) The Digital Universe of Opportunities: Rich Data and the Increasing Value of the Internet of Things. IDC Analyze the Future, 5.

Wolf, B. (2005) Brunswik's Original Lens Model. University of Landau, Germany, 9.

Zand, D. (1972) Trust and Managerial Problem Solving. Administrative Science Quarterly, 17(2), 229-239. doi:10.2307/2393957

Zarit, S. (2006) Assessment of Family Caregivers: A Research Perspective. In Family Caregiver Alliance. Caregiver Assessment: Voices and Views from the Field, 2, 12-37.

Zimmerman, J., \& Kurapati, K. (2002) Exposing Profiles to Build Trust in A Recommender. CHI Extended Abstracts on Human Factors in Computing Systems, Minneapolis, MN, April 2025, 608-609. ACM Press, New York. doi:10.1145/506443.506507 